\title{
STATISTIQUE ET BONHEUR DES HOMMES
}

On souhaiterait examiner ici la question suivante : sachant que d'Alembert, l'un des savants les plus profonds de son temps, vient de réduire la théorie ordinaire des probabilités à une œuvre de pure imagination, sachant que Turgot, l'un des administrateurs les plus éclairés de l'Ancien Régime, considère l'arithmétique politique du xvirie siècle comme fautive et dépourvue de toute valeur pratique, comment expliquer que Condorcet, ami et disciple de l'un et de l'autre, en soit arrivé à "promettre" au genre humain, dans la dixième période de l'Esquisse d'un tableau historique des progrès de l'esprit humain', qu'on parviendra à une précision presque mathématique dans "l'art social " en lui " appliquant le calcul des combinaisons et des probabilités".

On tentera de montrer, qu'en dépit d'apparences contraires, la démarche de Condorcet, loin d'être incohérente, représente un essai de synthèse, entre science et philosophie, unique au siècle des Lumières, qui permet à son auteur de proposer une codification raisonnée du comportement de l'homme en société, la "conduite de la vie ", laquelle n'est pas sans rappeler certaines idées développées dans la première moitié de ce siècle, auxquelles la statistique contemporaine continue d'adhérer pour une part.

Un grand nombre de savants ont déjà traité de questions semblables, aussi ne prétend-on nullement ici à l'originalité, tout a déjà été écrit et mieux qu'on ne saurait le faire. Mais la répétition des mêmes jugements n'est-elle pas le seul moyen de parvenir à la certitude, du moins à la seule certitude à laquelle nous puissions atteindre? Et si d'aventure il se trouvait au milieu de tant de constance quelque nouveauté ou quelques contradictions, ce serait simple inadvertance, qui ne devrait pas notablement affaiblir la probabilité de l'ensemble de la

I. Ed. Michèle et François Hincker, Paris, Editions sociales, 1971, p. 271. 
théorie et dont on voudra bien, pour cette raison, ne pas nous tenir rigueur.

\section{I. - POSITION DU PROBLEME}

Pour introduire notre propos, citons une fois encore le célèbre commencement du "Discours préliminaire " de l'Essai sur l'application de l'analyse à la probabilité des décisions rendues à la pluralité des voix ${ }^{2}$ :

«Un grand homme, dont je regretterai toujours les leçons, et surtout l'amitié, était persuadé que les vérités des Sciences morales et politiques, sont susceptibles de la même certitude que celles qui forment le système des Sciences physiques, et même que les branches de ces Sciences qui, comme l'Astronomie, paraissent approcher de la certitude mathématique.

Cette opinion lui était chère, parce qu'elle conduit à l'espérance consolante que l'espèce humaine fera nécessairement des progrès vers le bonheur et la perfection, comme elle en a fait dans la connaissance de la vérité ${ }^{3}$.

Il ne faut pas se méprendre sur les propos du grand homme dont il s'agit. Turgot, qui avait suivi au collège du Plessis les leçons de l'abbé Sigorgne 4 , propagandiste combatif du newtonisme en France, avait pris la mesure de l'abìme qui séparait l'Astronomie physique, la plus parfaite de toutes les sciences, des sciences morales et politiques les plus solidement établies sur les « vrais principes du droit naturel ", dont il connaissait si bien les limites qu'il se garderait de les mettre trop à l'épreuve, lorsqu'il aurait le pouvoir en main, leur préférant les quelques principes d'économie publique communs aux rares intendants éclairés du temps : liberté du travail, de la circulation des grains,

2. Paris, 1785, rééd. New York, Chelsea, 1972. On abrégera le titre en Essai et le « Discours préliminaire " en « Discours».

3. "Discours", p. i. Rappelons incidemment que, le propre d'une science étant d'être perfectible, il n'est nullement évident a priori que la morale et la politique puissent être objet de sciences, on est même parfois tenté d'en douter ; sur ce point, Augustin A. CourNOT, Considérations sur la marche des idées, Paris, Hachette, 1872, CEuvres complètes, Paris, Vrin, 1973, t. IV, p. 325 sq.

4. Pierre Sigorgne (1719-1809) a écrit notamment Examen et réfutation des leçons de physique expliquées par $M$. de Molières au Collège Royal de France, Paris, 1741. Turgot resta lié avec l'abbé Sigorgne jusqu'à sa mort, voir Alfred NeYMARCK, Turgot et ses doctrines, Paris, 1885 , t. II. 
suppression des douanes intérieures, des corvées, etc. ${ }^{5}$. Turgot, comme d'ailleurs d'Alembert, n'était pas tendre pour les «abus de science " qu'ils soient moraux ou physiques ; à vingt et un ans, déjà, il avait estimé devoir rappeler à l'ordre Buffon qui s'était autorisé certaines audaces cosmologiques dans sa théorie de la terre ${ }^{6}$ :

"Voulez-vous faire perdre à la philosophie de Newton cette simplicité et cette sage retenue qui la caractérisent? Voulez-vous, en nous replongeant dans la nuit des hypothèses, justifier les cartésiens sur leurs trois éléments et sur leur formation du monde? "'

Ce n'est assurément pas parce que les sciences morales et politiques sont susceptibles d'utiliser plus ou moins adroitement la langue du calcul qu'elles acquièrent la même certitude que les sciences physiques mais bien, comme l'écrit Condorcet, parce que le système des vérités qui forme les unes et les autres est de même nature, et relève de la même certitude.

La question qu'il s'agit d'examiner au préalable est donc celle de la nature de cette commune certitude.

Lorsqu'on lit superficiellement les réflexions de Condorcet sur le thème de la certitude, qu'on trouve disséminées dans toute son œuvre, on est frappé de leur peu d'originalité apparente. Comme pour la plupart de ses contemporains ${ }^{8}$, il n'existe pour Condorcet qu'une " certitude absolue ", celle " qui n'a lieu que pour les sciences abstraites, ou que dans les autres sciences pour la légitimité des conséquences qu'on

5. Il existe de très nombreux textes montrant la défiance de Turgot à l'égard des théories économiques trop ambitieuses, cf. par ex., 7e lettre à Terray, in Euvres de Turgot, éd. Gustave SChElle, Paris, Alcan, 1913-1923, t. III, p. 328 ; quant à l'arithmétique politique dont certains de ses collègues administrateurs s'étaient entichés, il a constamment dénoncé ses imprécisions et ses erreurs, cf. Lettre de Turgot à Price, 22 août 1780, in Henri Laboucheix, Richard Price, Paris, Marcel Didier, 1970, p. 33, dans laquelle Turgot répondait à Price qui venait de lui adresser son Essay on the Population of England, 1780 : «On manque presque partout d'éléments suffisamment exacts pour constater les faits, et on est peut-être encore plus loin de connaître l'influence des différentes causes qui concourent à faire varier ces faits. Je ne suis pas au reste éloigné de penser que cette science approfondie serait plus intéressante pour les philosophes qu'importante pour les politiques..."

Condorcet n'était d'ailleurs pas loin de penser la même chose à la même époque (avant 1780), voir par ex. sa polémique avec Moheau de 1778, Mercure de France, 5 juil. 1778, p. $35-41$ et 5 nov. 1778 , p. 66-67.

6. Buffon, Histoire naturelle, Paris, 1749, t. I, p. 130 sqq.

7. Turgot, Lettre à Buffon, sur la Théorie de la terre, oct. 1748, in Euvres.., op. cit. supra n. 5, t. II.

8. Cf. par ex., BUfFon, Essai d'arithmétique morale, in Histoire naturelle, Supplément IV, Paris, $1777, \S$ II, III. 
tire d'un principe supposé donné " 9 . Quant aux "certitudes physiques ou morales des Écoles " ${ }^{10}$, "ce ne sont que différents degrés de probabilité " ".

On ne compte plus les auteurs qui au XvIII e siècle ont écrit de semblables affirmations, chargeant le calcul des probabilités de suppléer aux faiblesses de la métaphysique dont les principes les plus clairs ou les mieux assurés, déracinés une fois encore, dérivaient doucement au gré des courants.

Rappelons par exemple que l'Académie de Berlin, inquiète à juste titre, avait cru devoir soumettre à son concours de 1763 les doutes de Hume sur la liaison des effets aux causes, en posant la question : "Les vérités métaphysiques sont-elles susceptibles de la même évidence que les vérités mathématiques, et quelle est la nature de cette certitude?" 12 .

Moses Mendelssohn avait remporté le premier prix devant Kant qui dut se contenter d'un accessit ${ }^{13}$. Il appartenait à l'école de Leibniz et Wolf qui, depuis un demi-siècle, s'employait à développer une logique du vraisemblable et qui dominait alors sans partage l'Académie de Berlin ${ }^{14}$.

Reprenant l'exemple du pain de Hume ${ }^{15}$, Mendelssohn écrivait :

« Nous croyons donc à l'effet qu'un aliment va produire sur la restauration de nos forces, par cette raison que si cet effet n'était pas lié à cet aliment, il eût été invraisemblable qu'il se fût répété si constamment, et tel est le lien qui unit l'expérience future à l'expérience passée. L'expérience passée nous fournit la probabilité d'une connexion secrète entre les phénomènes, l'expérience future en est l'application "16.

9. Hist. Acad. Sciences pour 1778, Paris, 1781, p. 46

10. Encyclopédie, article "Certitude", introduction de DIDEROT qui suivait mot à mot l'Encyclopédie de Chambers, 1728.

11. "Mémoires de mathématiques et physique", Savants étrangers, Acad. sciences, Paris, 1774, t. VI, p. 18.

12. Christian Bartholmess, Histoire philosophique de l'Académie de Prusse, Paris, 1851 , t. II, livre 8 , chap. II.

13. "Dissertation qui a remporté le prix proposé par l'Académie royale des sciences et belles lettres de Prusse, sur la Nature, les espèces et les degrés de l'Évidence avec les pièces qui ont concouru ", Berlin, 1764.

14. Joseph Marie DE GÉRANDO, Histoire comparée des systèmes de philosophie relativement aux principes des connaissances humaines, Paris, 1804, t. II, p. 126-155.

15. Enquête sur l'entendement humain, Paris, Aubier ("Bibliothèque philosophique "), 1947, p. $78-80$.

16. Sylvestre-François LACRoIX, Traité élémentaire du calcul des probabilités, Paris, $1816,1822,1833$, no 103, utilise une citation semblable de Mendelssohn pour montrer (à tort) que ce dernier est un précurseur de Condorcet. 
Mendelssohn ne faisait que reprendre ou retrouver les travaux de philosophes ou de savants anglais du temps comme Hartley ${ }^{17}$, Moivre ou Bayes dont nous aurons l'occasion de reparler et qui, manifestement, se préoccupaient de questions analogues.

La France n'était pas en reste, qui s'adonnait depuis quelque temps déjà à la philosophie de la sensation. Condillac avait réussi à réduire le système de Locke à sa plus simple expression : toute connaissance dérive de nos sensations convenablement transformées. Le problème de la certitude ne se pose donc qu'en terme de degrés et Helvétius n'avait plus qu'à conclure :

«... si presque toutes nos vérités se réduisent à des probabilités, quelle reconnaissance ne devrait-on pas à l'homme de génie qui se chargerait de construire des tables physiques, métaphysiques, morales et politiques, où seraient marqués avec précision tous les divers degrés de probabilité, et, par conséquent, de croyance qu'on doit assigner à chaque opinion ?

L'existence des corps, par exemple, serait placée dans les tables physiques comme le premier degré de certitude; on y déterminerait ensuite ce qu'il y a à parier que le soleil se lèvera demain, qu'il se lèvera dans dix, dans vingt ans, etc. Dans les tables morales ou politiques, on y placerait pareillement, comme premier degré de certitude, l'existence de Rome ou de Londres, puis celle des héros tels que César ou Guillaume le Conquérant ; l'on descendrait ainsi, par l'échelle des probabilités, jusqu'aux faits les moins certains, et enfin jusqu'aux prétendus miracles de Mahomet, jusqu'à ces prodiges attestés par tant d'Arabes, et dont la fausseté cependant est encore très probable ici-bas, où les menteurs sont si communs et les prodiges si rares $" 18$.

17. David Hartley, Observations on Man..., 1749, trad. franç. par l'abbé JuRain, Reims, 1755 et par Roch-Ambroise SICARD, Paris, 1802, cf. surtout dans cette traduction, t. II, sect. II, p. 153 : « Un ingénieux ami m'a communiqué une solution [...] dans laquelle il a démontre que quand un événement est arrivé $p$ fois et a manqué q fois, le rapport primitif des causes de l'occurrence ou défaut d'événement doit dévier dans un certain degré, de celle de $\mathrm{p}$ à $\mathrm{q}$. Il paraît que lorsque le nombre d'essais est très grand, la déviation doit être très peu considérable ; ce qui prouve que nous pouvons espérer de déterminer les proportions, et, par degrés, toute la nature des causes inconnues par une observation suffisante de leurs effets. $"$

L'identification de cet " ami ingénieux » et l'interprétation de ce passage sont actuellement l'objet de spéculations de la part des historiens de la statistique, cf. Stephen M. STIGLER, The American Statistician, 37, 1983, p. 290-296 et Andrew W. F. EDWARDS, ibid., 40, 1986, p. 109-110, qui montrent la difficulté de bien saisir la pensée des auteurs de cette époque, qui utilisent les mêmes mots et les mêmes expressions en des sens parfois diamétralement opposés. Nous aurons l'occasion d'éprouver ici les mêmes difficultés.

18. Helvétius, De l'esprit, Paris, 1758, t. I, p. 8-12, note (e) sur le calcul des probabilités, cité également par S.-F. LACrolX, in op. cit. supra n. 16, dans le même but.

Rappelons que Turgot avait condamné avec force le livre d'Helvétius que Condorcet avait défendu, cf. Charles Henry, éd., Correspondance inédite de Condorcet et de Turgot, Paris, Charavay Frères, 1883. 
Proposer de graduer ses certitudes ou son scepticisme ne saurait donc être regardé comme une innovation au XVIII ${ }^{e}$ siècle qui reprenait d'ailleurs là une tradition philosophique millénaire qu'il ne faisait que repeindre aux couleurs de l'empirisme : « l'expérience passée ».

Si l'on quitte un instant les certitudes et leurs dégradés harmonieux pour "l'art social " et la «conduite de la vie ", on est frappé des mêmes observations, Condorcet répétant partout que «ce n'est au fond qu'une suite de problèmes du calcul des probabilités ${ }^{19}$, et son siècle tout entier à l'exemple des siècles passés recommandant la prudence probabilisée.

Dans les tribunaux et les confessionnaux, on énumère et on compte les probabilités ${ }^{20}$. Les chemins du salut, terrestre ou éternel, sont jalonnés de cas de conscience aux issues incertaines. Qu'on choisisse la voie " la plus probable », comme l'a recommandé Rome à plusieurs reprises et même le Parlement de Paris en $1762^{21}$ ou qu'on se contente d'un parcours moins probable mais aussi moins escarpé, comme le tolèrent certains directeurs " doux » et avisés ${ }^{22}$, on s'en remet aux probabilités : « dans la pratique prudemment et probablement sont sinonimes $"{ }^{23}$.

Certes, les probabilités des métaphysiciens allemands, des philosophes français ou anglais, des juristes et des canonistes ne sont généralement pas assujetties aux règles fixes d'une analyse rigoureuse, ou si peu qu'on ne s'en aperçoit guère. Il y a toutefois de notables exceptions ${ }^{24}$ et certains auteurs n'hésitent pas à en appeler au calcul des chances des mathématiciens qui, du reste, les y encouragent par certaines ambiguïtés de vocabulaire et parfois même un excès de prétentions ${ }^{25}$.

19. Savants étrangers, op. cit. supra n. 11, p. 18-19.

20. La littérature sur ce sujet est très abondante, on se reportera à Ernest CoumET, Annales. Economies, Sociétés, Civilisations, 1970, p. 574-598 et Lorraine DASTON, Historia mathematica, 7, 1980, p. 234-260.

21. Jean MARIN, Extraits des assertions dangereuses et pernicieuses en tout genre... soutenues et enseignées par les soi-disants jésuites, Paris, 1762.

22. Ibid., p.9-105, qui cite 57 théologiens probabilistes entre 1600 et 1759, cf. TRACHALA, Lavacrum conscienciae, Bamberg, 1755: "Tout homme peut s'attacher à un motif gravement probable, sans se mettre en peine d'un motif opposé qui serait également ou plus probable. " Dans les Élémens du calcul des probabilités, Paris, 1805, p. 93, CoNDORCET justifie le probabilisme comme règle de conduite à l'aide de sa théorie de l'espérance contre Innocent XI et les dictionnaires des cas de conscience (celui de Pontas, 1715, ou de Lamet et Fromageau, 1733), il est vrai que ceux-ci conduisaient à la vie éternelle alors que Condorcet avait moins d'ambition ou en avait davantage encore.

23. Jean MARIN, op. cit. supra n. 21, p. 71.

24. C'est le cas notamment de la probabilité des témoignages, sur ce point on consultera l'article récent de S. M. STIGLER, Journal of the American Statistical Association, 81 , 1986, p. $879-887$.

25. L'exemple le plus célèbre étant l'Ars conjectandi de Jacques BerNoull, édition posthume, Bâle, 1713. 
Les philosophes les plus scrupuleux, conscients de l'enjeu, se livreront à une énumération attentive des sources de probabilités ${ }^{26}$, de celles nées des chances ${ }^{27}$, comme dans les jeux de hasard, à celles déduites de "l'expérience passée », de l'analogie, des témoignages ou de l'autorité d'un « docteur grave »; ces probabilités interviennent sur l'esprit humain comme autant de "forces", de "vigueur » variable, capables d'entraîner la conviction ou la croyance ${ }^{28}$. Par souci de généralité, on gardera au mode probabiliste de production de croyance une latitude suffisante pour englober toute la diversité des applications.

Sans entrer dans trop de détails, rappelons quelques exemples célèbres. Lorsqu'il s'agit de s'assurer de l'existence d'une cause à partir d'observations répétẻes d'un même phénomène, le raisonnement le plus fréquemment rencontré consiste à montrer le " ridicule " ${ }^{29}$ qu'il y aurait à en supposer l'absence, assimilée à un " pur hasard ", dont il est aisé de montrer par la doctrine des chances qu'il ne pourrait produire les événements observés, qu'avec une probabilité d'une extrême petitesse. Ce ridicule n'est généralement pas affecté d'un coefficient numérique bien précis, mais on rencontre parfois d'intéressantes formules d'inversion. Citons par exemple Buffon qui souhaite montrer que le mouvement des planètes du système solaire (6 à l'époque) n'est pas dû au hasard mais à une cause (pour Buffon la chute d'une comète sur le soleil) :

"Cette idée sur la cause du mouvement d'impulsion des planètes paraîtra moins hasardée lorsqu'on rassemblera toutes les analogies qui y ont rapport et qu'on voudra se donner la peine d'en estimer les probabilités. La première est cette direction commune de leur mouvement d'impulsion, qui fait que les 6 planètes vont toutes d'Occident en Orient; il y a déjà 64 à parier contre un qu'elles n'auraient pas eu ce mouvement dans le même sens, si la même cause ne l'avait pas produit, ce qu'il est aisé de prouver par la doctrine des hasards.

26. Par ex., David Hume lui-même, Traité de la Nature humaine, Paris, Aubier, 1946, t. 1, p. 205-239 (sur ce point of. Keith M. BAKER, Condorcet. From Natural Philosophy to Social Mathematics, Chicago, University of Chicago Press, 1975), ou BuffoN, op. cit. supra n. 6, p. 62 ou Essai d'arithmétique morale, op. cit. supra n. $8, \S$ IV, V, ou encore l'Encyclopédie, article « Probabilités».

27. Rappelons qu'en calcul des probabilités, le mot « chance » ou " hasard » prend un sens particulier, celui de " cas également possible » ; par ex., si l'on jette un dé « homogène ", chaque face a "une chance " de se produire. Les auteurs considèrent généralement ces chances comme données a priori (sans qu'on sache d'ailleurs très bien comment), le calcul des probabilités devient alors exact si l'on connaît ses règles.

28. C'est par exemple la théorie de D. Hume, in op. cit. supra n. 26 et celle de D. HARTLEY, in op. cit. supra n. 17.

29. Daniel Bernoulli, Recueil des pieces..., Acad. Sciences, Paris, 1734, t. III, p. 95-122, à propos des plans d'orbite des planètes. 
Cette probabilité augmentera prodigieusement par la deuxième analogie qui est que l'inclinaison des orbites n'excède pas 7 degrés et demi, car en comparant les espaces, on trouve qu'il y a 24 contre un que 2 planètes se trouvent dans des plans plus éloignés et par conséquent $24^{5}$ ou 7692624 à parier contre un, que ce n'est pas par hasard qu'elles se trouvent toutes 6 ainsi placées et renfermées dans l'espace de 7 degrés et demi, ou, ce qui revient au même, il y a cette probabilité qu'elles ont quelque chose de commun dans le mouvement qui leur a donné cette position ${ }^{30}$.

Conclusion intéressante, mais qui ne résulte plus de la doctrine des hasards, telle qu'on la pratique ordinairement. D'autres auteurs ${ }^{31}$ tenteront de semblables déductions qu'on peut parfois rendre correctes en explicitant les principes dont elles sont involontairement tirées ${ }^{32}$.

Buffon qui, en réalité, ne croyait à rien qu'en ses propres idées, n'est pas avare de formules " exprimant en nombres " les certitudes physiques ou morales dont nous sommes affectés; pour mesurer la certitude que le soleil se lèvera demain, on pourra raisonner ainsi :

“ Si l'on veut réduire ici l'ancienneté du monde et de notre expérience à six mille ans, le soleil ne s'est levé pour nous que 2 millions 190 mille fois; et comme, à dater du second jour qu'il s'est levé, les probabilités de se lever le lendemain augmentent, comme la suite $1,2,4,8,16,32,64 \ldots$ ou $2^{n-1}$, on aura (lorsque, dans la suite naturelle des nombres, $n$ est égal à 2190000 ), on aura, dis-je, $2^{n-1}=2^{2189099}$; ce qui est déjà un nombre si prodigieux que nous ne pouvons nous en former une idée... " ${ }^{33}$.

30. BufFon, Histoire naturelle, 1749, t. I, p. 139.

31. La littérature est très abondante, on pourra consulter, par ex., Willem Jacob s'GrAVESANDE, Euvres philosophiques et mathématiques, Amsterdam, 1774, t. II, p. 221-248, sur la masculinité, ou MAUPERTUIS, Lettre 14 "Sur la génération des animaux ", 1752, in Euvres, Lyon, 1756, t. II, p. 307-308, à propos de la transmission du sexdigitisme, ou DioNis DU SEJOUR, Traité analytique des mouvemens apparens des corps célestes, Paris, 1789, t. II, p. 345, à propos du mouvement des planètes. On trouve un énoncé analogue, quoique moins net, chez John MichelL, Philosophical Transactions, LVII, part I, 1768, p. 249, qui fut intensément discuté en Angleterre vers 1850.

32. Le raisonnement de Buffon s'écrit ainsi : si E désigne l'événement observé (les 6 planètes ont des plans d'orbites resserrés) et $C$ la cause dont l'absence, non $C$, est le hasard pur, on a $P($ non $E /$ non $C)=p$ ou «ce qui revient au mème » $P(C / E)=p$.

Si l'on admet le «principe de Condorcet " (voir § II) et si l'on suppose p proche de 1 ce qui est le cas ici, ce raisonnement est correct : la cause $\mathrm{C}$ impliquant nécessairement l'événement $E$, on a $P(E / C)=1$ et comme la probabilité d'une cause tirée de l'observation est proportionnelle à la probabilité de l'observation lorsque la cause en question agit, on $\mathrm{P}(\mathrm{C} / \mathrm{E})=\frac{1}{1+(1-\mathrm{p})} \simeq \mathrm{p}$.

33. Essai d'arithmétique morale, op. cit. supra n. $8, \S \mathrm{VI}$, voir aussi $\S \mathrm{X}$. 
Il faudrait naturellement parler ici de l'Essay de Bayes qui est de très loin la tentative la plus intéressante, avant Condorcet et Laplace, pour réduire en nombres nos probabilités et nos certitudes, mais le sujet a été fort bien traité par d'autres, auxquels nous renvoyons ${ }^{34}$. Il semble d'autre part établi ${ }^{35}$ que le mémoire de Bayes et Price a été reçu en France seulement après que Turgot puis Condorcet soient entrés en rapport avec Price, vers 1778 , et que Condorcet et Laplace aient entrepris, indépendamment, de développer la statistique mathématique. Mais n'anticipons pas.

Les probabilités déduites des témoignages obéissent à des règles plus souples et variables suivant les auteurs ${ }^{36}$. Quant aux probabilités tirées des analogies, la doctrine des hasards n'étant plus d'un grand secours, on se contentera de généralités encore plus flexibles ${ }^{37}$.

On aurait pu assurément s'en tenir à une position de principe et ne retenir de toutes ces considérations numériques que celles déduites directement de la théorie ordinaire des chances fondée depuis plus d'un siècle sur l'autorité des mathématiciens les plus habiles ; encore eût-il fallu qu'ils fussent unanimes, le témoignage d'un seul « docteur grave " pouvant en l'occurrence rendre incertaine toute conclusion et

34. Thomas BAyes, "An Essay towards Solving a Problem in the Doctrine of Chances ", Phil. Trans., 53, 1764, p. 370-418, trad. franç., notes et commentaires, JeanPierre Cléro, Cahiers d'histoire et de philosophie des sciences, à paraître. Du même auteur, on lira les "Remarques philosophiques sur l'Essai de Bayes », Publications de I'I.R.M.A.R., Rennes I, 1986, no 2, p. 1-32.

On se reportera également à un article très complet de DonALD A. Gillies, à paraître, dans Historia mathematica, qui donne une bibliographie détaillée, et au livre de $S$. M. Stigler, The History of Statistics, Harvard University Press, 1986, p. 9498.

35. Il est de notoriété publique que CONDORCET a cité le mémoire de Bayes pour la première fois en 1781 dans Hist. Acad. Sciences pour 1778, p. 46-47, D'ALEMBERT l'ayant précédé de peu dans ses Opuscules VII en 1780 . Il faudrait pour augmenter la probabilité de ces affirmations reprendre toute la correspondance de Price dispersée en divers endroits, cf. H. Laboucheix, op. cit. supra n. 5, ce qui ne semble pas avoir été fait à l'heure présente. La question n'est pas simple à trancher puisque les Philosophical Transactions étaient relativement bien connues des savants du Continent, au moins par les extraits publiés en français dans certains journaux, par ex. le Journal encyclopédique, qui en 1765 et 1766 publia le résumé des volumes contenant les deux articles de Bayes et Price, où l'on peut lire (Journal encyclopédique, 15 févr. 1765, t. II (1), p. 22) : " Essai sur la solution d'un problème concernant la doctrine du hazard. C'est là le titre de l'article 52e par M. Price, et auquel on nous dispensera de nous arrêter. " Et pour le mémoire de Price publié l'année suivante (Phil. Trans., LIV, 1764) : « Le 52e article est une démonstration de la $2 e$ règle des essais de la solution d'un problème dans la doctrine du hazard, publié dans le 43 e volume des Transactions Philosophiques par Richard Price. On est bien étonné de trouver dans cette collection, un article rempli d'absurdités. Les plus grands Algébristes avouent de bonne foi, qu'ils n'y comprennent rien " (Journal encyclopédique, 15 janv. 1766 , t. I (2), p. 84 ).

36. Cf. supra $\mathrm{n} .24$.

37. Là encore BUfFON est remarquable, Essai d'arithmétique morale, $\S$ VII et VIII, qui permettent de comprendre l'hostilité fondamentale qu'éprouvaient pour lui d'Alembert et Condorcet (et réciproquement). 
la soumettre à son tour à une difficile évaluation de probabilité. Or d'Alembert, qui ne tolérait pour les sciences que des principes clairs et distincts, qui bannissait toute métaphysique et que l'idée même qu'on puisse la remplacer par un calcul de probabilités devait irriter au plus haut point, se livrait depuis 1750 à une entreprise de destruction systématique non seulement des conclusions directes ou inverses qu'on tirait ordinairement de la théorie des hasards mais aussi des règles et des principes premiers sur lesquels ladite théorie était fondée ${ }^{38}$.

D'Alembert ne niait évidemment pas la nécessité où l'on était de conjecturer en situation d'incertitude mais il doutait qu'on puisse jamais en faire l'objet d'un calcul : "le calcul n'a pas de prise suffisante $"{ }^{39}$. Conjecturer est un art qui se confond avec "l'art de se conduire " et " le plus habile dans cet art est celui dont les conjectures sont le moins souvent démenties par les faits $"{ }^{40}$.

Le calcul des probabilités que l'on voulait faire servir à mesurer le degré de nos certitudes physiques ou morales et qui devait éviter à la philosophie de la sensation d'être aspirée par le gouffre du pyrrhonisme où elle perdait son âme, serait-il aussi peu fondé que les principes métaphysiques que l'on avait cru bon d'abattre? Pourtant, la théorie ordinaire des hasards, tout le monde en convenait, reposait sur un seul " principe général " suffisamment simple et clair pour " assurer qu'il donne au moins un assentiment pratique à toute la doctrine " ${ }^{41}$, à savoir, comme le rappelle Condorcet ${ }^{42}$ :

" Si sur un nombre donné de combinaisons également possibles, il y en a un certain nombre qui donnent un événement, et un autre nombre qui donnent l'événement contraire, la probabilité de chacun des deux événements sera égale au nombre des combinaisons qui l'amènent, divisé par le nombre total. "

Encore fallait-il s'entendre sur les combinaisons qu'il était légitime ou naturel ${ }^{43}$ de déclarer " également possibles ".

38. Sur les doutes de d'Alembert, cf. Michel PATY, « D'Alembert et les probabilités », in Sciences à l'époque de la Révolution française..., éd. Roshdi RASHED, à paraître.

39. D'AlEmBERT, " Doutes et questions sur le calcul des probabilités », Mélanges de littérature, d'histoire et de philosophie, t. V, 1767, p. 289.

40. Éclaircissement des éléments de philosophie, VI, in Euvres, Paris, 1805, t. II, p. 121.

41. D. HARTLEY, op. cit. supra n. 17, p. 151.

42. "Discours», p. v.

43. On faisait généralement dépendre l'égale possibilité en question soit de considérations physiques, l'homogénéité, l'égale propension, etc., prises dans la nature des choses, soit de conditions juridiques, règlements équitables d'un jeu, d'un pari... D'Alembert contestait les premières aussi bien que les secondes. 
" Pascal, Jacques et Daniel Bernoulli, Moivre, etc. étaient grands géomètres et gens de beaucoup d'esprit. Ils perfectionnèrent la doctrine des combinaisons ou plutôt ils la créèrent et leurs méthodes furent si ingénieuses, leurs conclusions si élégantes et leurs résultats si piquants et si plausibles que personne ne s'avisa de contredire leurs principes encore moins de les examiner $» 44$.

Il n'y avait rien de choquant à fonder une science sur l'ingéniosité de ses créateurs et le piquant de ses résultats, à condition de ne pas lui attribuer un " sens étendu » qui règle le système de nos certitudes. D'autant que la science dont il s'agissait était contestée par un savant, d'Alembert, qui pouvait rivaliser d'esprit et de géométrie avec les grands noms dont elle se recommandait.

Or Condorcet, comme Turgot (et d'Alembert), ne se souciait pas seulement d'ingéniosité, ni même d'utilité mais principalement de vérité et de justice, fondements nécessaires de la morale et de la politique $^{45}$ et de « la science la plus intéressante de toutes, celle du bonheur public ${ }^{46}$.

Pour probabiliser les certitudes physiques ou morales et répondre aux vœux de Turgot ${ }^{47}$, il fallait commencer par faire taire la cacophonie probabiliste où l'on ne s'entendait plus et fonder sur des bases véritables et justes les probabilités alors en question.

Il n'est pas si facile de suivre l'évolution des réflexions de Condorcet sur ce problème, jetées au hasard de ses innombrables écrits publiés ou non ${ }^{48}$. De l'avis de Condorcet lui-même ${ }^{49}$, le texte de référence est le «Discours préliminaire » de 1785 auquel il faut associer une partie de l'Éloge de Turgot publié en 1786 et rédigé probablement dans le même temps.

Ces deux textes semblent avoir fixé la pensée de Condorcet qui ne se modifiera plus sensiblement ainsi qu'en témoignent ses dernières œuvres, l'Esquisse ou les Éléments du calcul des probabilités.

Si l'on veut tenter de comprendre l'originalité et la profondeur de la

44. Condorcet, Paris, Institut, ms 883, ff. 216-217.

45. « Discours», p. iii.

46. Lettre de Turgot à Price, 22 mars 1778, in Honoré-Gabriel, comte DE MirABEAU, Considérations sur l'ordre de Cincinnatus, Londres, 1784, p. 185-203.

47. Turgot dans sa correspondance avec Condorcet parle de la probabilité d'une théorie, il se proposait d'ailleurs d'écrire les articles « Certitude " et " Probabilité " de l'Encyclopédie (voir la fin de l'article « Existence », 1756, t. VI), qui nous auraient certainement éclairé davantage sur ces questions. On peut penser que Condorcet voulut suppléer le grand homme défaillant.

48. On attend avec impatience la publication intégrale et commentée des manuscrits probabilistes entreprise à l'initiative et sous la direction de Pierre CrÉPEL.

49. Institut, ms. 875, ff. 179 : " J'ai prouvé dans l'ouvrage [le « Discours... "] pages v et suivantes que ce rapport était la véritable expression de la probabilité... » 
doctrine des probabilités de Condorcet, il faut néanmoins commencer par rappeler brièvement son itinéraire probabiliste tel qu'on peut le saisir actuellement, en espérant que d'autres manuscrits ou d'autres découvertes viendront confirmer ou plutôt infirmer, comme nous l'espérons, les conjectures et les interprétations qu'on a dû leur substituer. La critique des textes ne procède, on le sait, que par négations successives.

\section{II. - UN ITINÉRAIRE PROBABILISTE ${ }^{50}$}

Tout commence en cet été de 1772 que Condorcet passait, comme à son habitude, dans la maison maternelle de Ribemont, où il "s'amusa " à calculer des probabilités ${ }^{51}$. Il n'est pas interdit de penser que ce nouvel intérêt de Condorcet pour une doctrine d'ailleurs contestable était né au printemps de la même année après qu'il eût entendu à l'Académie le premier mémoire de Laplace " sur les séries récurrorécurrentes ", lu le 5 février, et appris par le Journal astronomique de Jean (III) Bernoulli que Lagrange et Daniel Bernoulli s'intéressaient à la recherche du milieu qu'il faut prendre entre plusieurs observations, version astronomique de l'identification des causes par les effets ${ }^{52}$. La rédaction de ces amusements probabilisés se trouve aux archives de l'Institut et du Bureau des longitudes ${ }^{53}$; il s'agit d'une

50. L'auvre probabiliste de Condorcet a été très bien étudiée, on se reportera à K. M. BAKER, Condorcet... op. cit. supra n. 26; P. CRÉPEL, "Condorcet, la théorie des probabilités et les calculs financiers ", à paraître dans Sciences à l'époque de la Révolution française, op. cit. supra $\mathrm{n}$. 38 et "Condorcet et l'estimation », à paraître dans le Journal de la Société de statistique de Paris; Gille-G Granger, La Mathématique sociale du marquis de Condorcet, Paris, P.U.F., 1956 ; R. RASHED, Condorcet. Mathématique et société, Paris, Hermann, 1974 ; Karl Pearson, The History of Statistics in the $17^{\text {th }}$ and $18^{\text {th }}$ Centuries, E. S. Pearson, éd., 1978 ; Isaac TodhunTer, $A$ History of the Mathematical Theory of Probability, 1865, New York, Chelsea, 1949 ; et divers articles d'Oscar B. SHEYNIN dont la liste et l'index figurent dans Archive for History of Exact Sciences, 28 (2), 1983, p. 171-195.

51. Correspondance inédite de Condorcet et de Turgot, op. cit. supra n. 18, p. 97. Cf. K. M. BAKER, op. cit. supra n. 26.

52. Sur la chronologie des æuvres probabilistes de Laplace, cf. Charles C. GiLlisPIE, article « Laplace » du D.S.B. et S. M. STIGLER, Isis, 69, 1978, p. 234-254 ou The History of Statistics, op. cit. supra n. 34, p. 102.

On peut, si l'on préfère les causes psychologiques, invoquer aussi le désespoir amoureux. Condorcet se mourait alors d'amour pour Madame de Meulan qui s'en divertissait, si l'on en croit Janine Bouissounouse, Condorcet, le philosophe dans la Révolution. Paris, Hachette, 1962.

53. On partira ici de l'hypothèse que le manuscrit reconstitué par $P$. Crépel : Institut, ms. 883, ff. 216-221, Bureau des longitudes, $Z 30$ ff. 1-6, Institut, ms. 875, ff. 132-133, est convenablement daté de l'été 1772 , suivant l'opinion de plusieurs docteurs dont la gravité n'est plus à démontrer. Sur ce point, on se reportera à P. Crépel, Historia mathematica, 14, 1987. 
réflexion libre sur les doutes de d'Alembert dans laquelle Condorcet se persuade que le calcul ordinaire des probabilités peut, lorsqu'il est ramené à une " mesure commune" - tirer une boule "d'un nombre $\mathrm{m}+\mathrm{n}$ de boules, $\mathrm{m}$ blanches et $\mathrm{n}$ noires" - , et qu'on interprète convenablement ses résultats, permettre d'évaluer la «valeur moyenne " d'un jeu, la " certitude qu'on peut avoir " de la composition d'un sac de boules dont on a observé les résultats de plusieurs tirages (c'est-à-dire la certitude des résultats de l'arithmétique politique : tables de mortalité, taux de natalité, etc.), et même "la probabilité qu'une chose qui a un dessein a un ouvrier".

La victoire serait-elle acquise sans combats et le but atteint avant même qu'on soit parti ? De telles situations sont fréquentes en histoire des sciences, où l'on commence parfois par arriver, la difficulté étant de s'en convaincre et d'en convaincre les autres. Pour être plus clair, il faut entrer dans quelques détails et reprendre point par point le manuscrit dont il s'agit.

La première question abordée par Condorcet est celle du sens qu'il faut attribuer aux "valeurs moyennes " ou " espérances " calculées par la doctrine en usage, si l'on veut élucider, notamment, le " problème des mémoires de Petersbourg " ${ }^{54}$. Condorcet propose une théorie originale qu'il ne publiera que douze années plus tard ${ }^{5 s}:$ la valeur moyenne (ou espérance) calculée par la règle ordinaire n'est pas la "valeur vraie " mais c'est la seule qui possède suffisamment de propriétés pour pouvoir en tenir lieu. En dépit du fait qu'elle devienne dans certaines circonstances réellement désavantageuse ${ }^{56}$, elle permet de réaliser, sur le long terme, un équilibre entre les parties, avec une probabilité sans cesse croissante.

Condorcet aborde ensuite le problème des applications du calcul des probabilités aux " événements de la vie ", lorsque " le nombre des effets possibles n'est connu que par l'expérience ", et que l'on est

54. Cf. par ex., M. PATY, art. cit. supra n. 38 et Gérard JoRLAND, in The Probabilistic Revolution, eds Lorenz Kruger, Lorraine J. Daston, Michael Heidelberger, Cambridge, Mas., M.I.T. Press, 1987, vol. I, p. 157-190.

55. "Mémoire sur le calcul des probabilités ", lre partie, Hist. Acad. Sciences pour 1781, Paris, 1784, p. 707-720. Condorcet a cependant annoncé sa théorie de l'espérance dès 1773 dans son "Éloge de Huygens", Euvres de Condorcet publiées par A. Condorcet $O$ 'Connor et $M$. F. Arago, Paris, 1847-1849, t. II, p. 64-65 et l'a répétée en de nombreux endroits, cf. en 1776 dans l'« Éloge de Pascal », ibid., t. III.

56. Par ex., si un joueur a de très faibles chances de gagner un enjeu important $A$ contre un adversaire qui aurait de fortes chances $b$ de gagner l'enjeu $B$ tel que $a A=b B$, le joueur ne devrait se « déterminer à jouer à ce jeu que dans l'espérance de jouer assez de coups " pour avoir une probabilité consistante de gagner au moins une fois la somme A, qui compenserait alors toutes les pertes, faibles mais répétées, qu'il a dû subir au préalable ; ce qui détruit à la fois l'argument du pari de Pascal (Éloge de Pascal, Avignon, 1776 , p. 47-49) et résout le problème de Petersbourg (cf. Bureau des longitudes, Z30 ff. 3). 
"dans le même cas qu'un homme qui aurait un sac de boules noires et blanches, ignorerait le nombre des unes et des autres et ne pourrait en juger que d'après le nombre qu'il aurait amené des unes ou des autres en tirant à diverses reprises une boule du sac $"{ }^{57}$, cette comparaison, "empruntée à Jacques Bernoulli ", permettant "d'établir les principes du calcul des probabilités sur cette matière ", la plus importante de toutes.

Le principe proposé par Condorcet, bien qu'il ne soit pas explicitement énoncé, est entièrement original, autant qu'on puisse le savoir; il deviendra bientôt sous la plume de Laplace ${ }^{58}$ :

"Si un événement peut être produit par un nombre n de causes différentes, les probabilités de l'existence de ces causes prises de l'événement sont entre elles comme les probabilités de l'événement prises de ces causes, et la probabilité de l'existence de chacune d'elles est égale à la probabilité de l'événement prise de cette cause, divisée par la somme de toutes les probabilités prises de chacune de ces causes."

On ne tranchera pas ici la question de savoir si Laplace a appris son principe de Condorcet, ou si Condorcet s'est servi d'un manuscrit non publié de Laplace, bien que cette seconde solution paraisse assez invraisemblable quand on connait les personnalités des protagonistes ${ }^{59}$.

Quoi qu'il en soit, ce principe que Condorcet semble considérer comme une extension naturelle du principe des cas également possi-

57. Bureau des longitudes, $Z 30$ ff. 3.

58. "Mémoire sur la probabilité des causes par les événements", CEuvres complètes de Laplace, Paris, Gauthier-Villars, 1891, t. VIII, p. 29. Ce mémoire a vraisemblablement été composé au printemps 1773 ; sur ce point, cf. S. M. STIGLER, op. cit. supra n. 34, chap. III.

59. Supposons comme Condorcet (Bureau des longitudes, $Z 30$ ff. 4) qu'on sache que le sac contient $2 \mathrm{n}$ boules noires ou blanches et "qu'ayant tiré $2 \mathrm{~m}$ coups " on ait "amené " $\mathrm{m}$ blanches et $\mathrm{m}$ noires, "le nombre de combinaisons qui donnent ce résultat " si l'on suppose que le sac contient $n$ boules blanches et $n$ boules noires est $C_{2 m}^{m} n^{m}$; ce nombre deviendrait $2 C_{2 m}^{m}(n+k)^{m}(n-k)^{m}$ si l'on supposait que le sac contient $n+k$ boules d'une couleur et $n-k$ de l'autre. D'où il résulte que la probabilité (prise de l'événement) qu'il y ait dans le sac autant de boules noires que de blanches est $n^{2 m} / 2 . \sum_{k=0}^{n-1}\left(n^{2}-k^{2}\right)^{m}$ et la probabilité que les nombres de boules ne s'éloignent de l'égalité que de $p$ est $\sum_{k=0}^{p}\left(n^{2}-k^{2}\right)^{m} / \sum_{k=0}^{n-1}\left(n^{2}-k^{2}\right)^{m},\left(^{*}\right)$.

Condorcet s'est arrêté là ; pour poursuivre, comme Laplace l'a fait (cf. op. cit. supra n. 58), il suffisait pourtant de supposer les nombres assez grands pour qu'on puisse assimiler le rapport $\mathrm{x}$ des boules blanches à toutes les boules du sac à un nombre compris entre 0 et 1 , la formule $\left(^{*}\right)$ devenant alors $\int_{1 / 2-a}^{1 / 2+a} x^{m}(1-x)^{m} d x / \int_{0}^{1} x^{m}(1-x)^{m} d x$, qui visiblement, pour tout a et lorsque $m$ est suffisamment grand, est aussi approchant de 1 qu'on le voudra, comme le montrera LAPLACE (Euvres complètes, op. cit. supra $\mathrm{n}$. 58, t. VIII, p. 33). 
bles permet, une fois mis en œuvre, de répondre de la vraisemblance des compositions possibles d'un sac empli de boules, considérées comme autant de causes, d'après la couleur des boules qu'on en a tirées ${ }^{60}$, et par conséquent de la probabilité des rapports déduits des états de population, baptêmes, mariages et sépultures ${ }^{61}$.

Reste la recherche de la probabilité d'une "cause intelligente " lorsque « se présente une suite d'événements qui paraît suivre une certaine règle et tendre à une fin ", par exemple si l'on trouve le mot ROMA «combien est-il probable que les lettres ont été arrangées pour former ce mot? $" 62$.

Il semble que Condorcet ait voulu appliquer le même principe ; sur les 24 permutations possibles des quatre lettres $R, O, M$ et $A, 9$ sont intelligibles (roma, ramo, oram, omar, omra, mora, maro, armo, amor ${ }^{63}$ ) et 15 ne le sont pas; emporté par son élan, Condorcet en conclut que « la probabilité que Roma a été écrite avec dessein ne sera donc que 9 à 15 ou 3 à 5 " alors qu'il aurait dû conclure à une probabilité de $8 / 11$ s'il avait suivi le principe de proportionnalité qu'il avait introduit, ainsi que le corrigera bientôt Laplace ${ }^{64}$ et que Condorcet en conviendra finalement ${ }^{65}$.

Cette coquille mise à part, le premier essai sur les probabilités de Condorcet, s'il est convenablement daté, est d'un intérêt exceptionnel et l'on peut s'étonner que Condorcet ne l'ait pas publié. Il est vrai qu'il ne s'arrête pas là et qu'il se propose également de traiter du "bonheur » ou du " malheur» au jeu. D'Alembert avait tenu sur ce thème des propos particulièrement anticonformistes, étayés par l'arti-

60. Pour tenter de se faire une opinion, on relira le commentaire de CoNDORCET relatif au mémoire de Laplace (op. cit. supra n. 11, p. 18-19), dans lequel Condorcet semble réduire l'apport de Laplace à l'idée d'introduire les " quantités différentielles ", idée qui, du reste, a déjà été exploitée par Daniel Bernoulli et d'Alembert, comme il le note incidemment. On lira aussi le ms. 855, ff. 157-158, dans lequel Condorcet présente Laplace à Turgot comme " un géomètre très fécond qui a porté l'application du calcul des probabilités à l'économie politique plus loin qu'elle ne l'a été jusqu'ici ".

61. Z 30 ff. 5 .

62. Z 30 ff. 6, il s'agit d'un exemple classique qui remonte au moins aux stoïciens (cf. CICÉron, De nat. deorum, II, XXXVII) et que Condorcet a emprunté à d'Alembert, "Doutes..." op. cit. supra n. 39, et Encyclopédie, article "Combinaison" qui reprenait l'Encyclopédie d'Ephraim Chambers, article "Change " qui reprenait John Wall.s, $A$ Discourse of Combinations..., in A Treatise of Algebra, Londres, 1685, chap. $11, \S 8$, etc.

63. Institut, ms. 875 , ff. 132 .

64. "Recherches sur l'intégration des équations", Savants étrangers, op. cit. supra n. 11, VII, 1776, Euvres complètes, op. cit. supra n. 58, t. VIII, p. 151-152; ce texte a sans doute été écrit en 1774, comme partie d'un " traité complet " de calcul des probabilités que Laplace n'aura le temps de compléter qu'en 1812.

65. Dans son «Mémoire sur le calcul des probabilités », 2e partie, op. cit. supra n. 55, p. 720-728.

Pour une discussion plus détaillée du « manuscrit de 1772 », on se reportera aux deux articles de P. Crepel cités supra $n$. 50. 
cle "Fatalité " de l'Encyclopédie ${ }^{66}$, auxquels Condorcet devait répondre ${ }^{67}$. Il ne semble pas en avoir eu le temps, puisque le manuscrit tourne court et se termine par un mystérieux "à revoir ", après avoir indiqué que « la théorie purement mathématique des combinaisons ne donne point ce qui doit arriver et que les événements dépendant de causes inconnues sont déterminés indépendamment de cette théorie ".

Parvenu au sommet, Condorcet s'est-il trouvé à son tour assailli de doutes comme ses plus illustres prédécesseurs, Jacques Bernoulli et Thomas Bayes, qui hésitèrent jusqu'à leur mort à publier leurs résultats et finalement renoncèrent? C'est un trait de caractère qu'il n'est pas si rare de rencontrer chez certains géomètres, trop habitués aux " certitudes absolues" pour se satisfaire aisément de "certitudes morales ", que cette sorte de paralysie dont ils sont frappés lorsqu'ils abordent les questions, touchant à la conduite de la vie et à la philosophie de la connaissance, que l'on se pose inévitablement à propos du calcul des probabilités ; de ce vertige probabiliste, maladie professionnelle d'ailleurs reconnue, Condorcet se gardera en pratiquant cette forme d'humour énigmatique qui lui est si particulière : tout cela n'est qu' " amusement ", " je ferai, écrit-il à Turgot le 3 septembre 1772, un petit livre sur cet objet d'où il résultera, à ce que j'espère, que nous savons bien peu de choses sur cette matière. Je suis au fond de l'avis de M. d'Alembert, et nous ne différons que dans quelques détails ${ }^{68}$.

Il existe plusieurs versions de ce "petit livre" dans les manuscrits de Condorcet qui parlera à nouveau en " 1774 " d'un " petit ouvrage plus métaphysique que mathématique " qu'il a sur les probabilités ${ }^{69}$. Ces textes s'échelonnent de 1772 à 1784 sans qu'on en sache établir une chronologie précise ${ }^{70}$. Ils sont tous empreints d'un fond de scepticisme qui peut expliquer que Condorcet ait hésité à les publier en l'état, d'autant que Laplace, pendant cette même période, a multiplié les mémoires dans lesquels, sans l'ombre d'un doute apparent, il a développé, suivant les " principes posés " (peut-être) par Condorcet, et à l'aide d'une analyse particulièrement déliée, une véritable statistique mathématique capable de réduire en nombres les vraisemblances des événements de la vie et des causes prises de ces événements ${ }^{71}$.

66. "Doutes... ", op. cit. supra n. 39, p. 301-304.

67. Laplace traitera bientôt ce même problème dans le mémoire cité supra n. 64 , p. 151 .

68. Lettre du 3 septembre 1772 , in op. cit. supra n. 51 .

69. Lettre à Turgot non datée, in ibid., p. 197 ; sur ce point, cf. K. M. BAKER et P. CréPEL, op. cit. supra n. 50.

70. Celle-ci fera l'objet d'une discussion aussi serrée que possible dans l'édition de P. Crépel des manuscrits probabilistes de Condorcet, à paraître.

71. Pour la chronologie des mémoires de Laplace, voir l'article "Laplace " du D.S.B., op. cit. supra n. $\mathbf{5 2}$. 
Condorcet, qui ne pouvait se contenter de solutions techniques et qui, de surcroît, se trouvait pris de court par la virtuosité analytique incomparable de Laplace, s'est donc tu, se limitant à quelques allusions sibyllines et incompréhensibles pour le profane, égrenées parcimonieusement dans ses "Éloges " et ses rapports académiques.

Nous nous limiterons ici à l'examen sommaire du seul de ces manuscrits dont on puisse penser qu'il était prêt à l'impression, puisqu'il n'est pas de la main de Condorcet mais de celle d'un copiste et sur grand papier ${ }^{72}$. 11 est probablement tardif, disons $1780 \pm 1$ avec probabilité $3 / 5$, pour fixer les idées.

Le manuscrit est en deux parties, l'une " historique », l'autre "métaphysique "; il se propose visiblement de résoudre une fois de plus les difficultés du calcul des probabilités.

Dans la partie historique, Condorcet présente à nouveau comme allant de soi le principe des cas également possibles pour un sac de boules de composition donnée et son « extension " aux " événements de la vie ". Par une faiblesse de caractère qui lui est propre, Condorcet ne s'attribue aucune part dans l'extension dont il s'agit, renvoyant à "Laplace " puis à "Bayes et Price ". Il explique ensuite que de ces principes clairs et distincts aurait dû résulter une théorie certaine et évidente, si quelques-uns de ses résultats n'avaient choqué le bon sens et si d'Alembert n'avait cru de son devoir de révoquer en doute le principe fondateur et tout l'édifice.

Condorcet remarque alors qu'il n'est pas nécessaire de modifier les principes, il suffit d'indiquer «la nature des vérités » auxquelles ils conduisent. C'est l'objet de la deuxième partie du mémoire.

Il ne faut pas se payer de mots, ces cas que l'on déclare également possibles ne le sont que dans notre esprit sans qu'ils ne le soient ni réellement ni même physiquement. Il s'agit d'une possibilité «abstraite ", d'une "considération purement intellectuelle ", ce qui du reste n'affecte ni le calcul lui-même, qui conserve toute sa cohérence, ni le piquant des résultats auxquels il conduit ${ }^{73}$. Avant tout calcul, il y a donc jugements et décisions préalables, or ces jugements de probabi-

72. Institut, ms. 875 , ff. $100-109$.

73. Sur ce point voir MASSE DE LA RUDELIÉRE, Défense de la doctrine des combinaisons..., Paris, 1763 , p. 96 . Il faudrait développer ici l'histoire du statut des objets mathématiques dont les liens éventuels avec les réalités du monde sensible sont conçus différemment suivant les époques. Pour la période contemporaine, on renverra au très beau texte de Jean CAVAllLES, «Du collectif au pari ", Revue de métaphysique et de morale, 1940, p. 151.

Il ne faut pas non plus prendre trop à la lettre l'idéalisme dont fait preuve ici Condorcet ; à de certains endroits, le moment venu, il considérera la probabilité comme un concept physique (cf. « Discours", p. xiv), sous le nom de " probabilité propre », prise dans la nature même des choses. 
lité peuvent légitimement être formés de plusieurs manières, suivant qu'on considère l'événement dont il s'agit, a priori, " métaphysiquement » ou dans des ensembles plus ou moins étendus d'événements de même nature.

Comment alors juger de nos certitudes, et régler notre conduite sur de telles "considérations de notre esprit, tout à fait étrangères à l'ordre des choses ${ }^{74}$ ? Suivent les rappels d'usage :

« Observons qu'à l'exception des vérités mathématiques, des propositions identiques de la métaphysique, et de celles qui expriment nos sensations actuelles, il n'y a aucune espèce de proposition qui soit certaine, que toutes sont probables, toutes appartenant à cette partie du calcul des probabilités où l'on juge de l'ordre futur des événements par celui qu'ils ont observé ou, de ce qui est plus précis, de l'ordre des événements inconnus par celui des événements connus ",

et l'on développera " cette vérité par des exemples tirés de différentes classes de connaissance " : "l'aimant attire le fer ", " un métal " possédant telle ou telle propriété "est de l'or ", "César a existé " et même " ce livre, que je vois, que je touche, existe", sont des propositions dont on peut calculer la probabilité par la règle de Bayes-PriceCondorcet-Laplace à partir d'observations ou de jugements répétés "qui ne se sont jamais démentis". Mais comme "on a prouvé que cette probabilité était absolument indépendante de l'état réel des choses, il [me] semble qu'il ne reste aucun moyen d'expliquer comment nous devons croire ce qui est probable et nous conduire d'après cette probabilité..." ".

Le mémoire s'interrompt là après quelques formules consolantes mais peu convaincues.

Ainsi Turgot avait raison, les sciences physiques et morales sont susceptibles des mêmes certitudes assujetties aux règles d'un même calcul, mais d'Alembert avait également raison, tout ce calcul est " abstrait " et ne saurait avoir de rapport avec l'ordre réel des choses ni permettre aucune prévision.

Qu'avaient alors à attendre la Vérité et la Justice d'un calcul qui permettait au mieux de répondre au vœu d'Helvétius qu'on dressât un tableau comparatif des certitudes et des incertitudes des connaissances humaines, sans qu'on puisse connaître la certitude du tableau lui-même ni le faire servir pour la conduite de la vie?

Comment fonder encore une morale et une politique justes et vérita-

74. Nous en sommes au folio $105 / 106$. 
bles? Autant se régler sur l'utilité et le plaisir comme Helvétius et d'autres.

Et pourtant, quelques années plus tard, Condorcet déclare solennellement dans son « Discours sur l'astronomie et le calcul des probabilités ", lu au Lycée en $1787^{75}$, qu'il sait distinguer ce qui est une proposition mathématique, un résultat du calcul, de ce qu'on peut regarder comme une vérité réelle et prouver que le motif de croire à ces vérités réelles se déduit véritablement d'un calcul de probabilités.

Entre-temps, Condorcet s'est enfin décidé à publier son " Mémoire sur le calcul des probabilités " en plusieurs parties ${ }^{76}$, de 1784 à 1787 , qui reprennent l'essentiel du " manuscrit de 1772 » en le développant considérablement. Et surtout le « Discours préliminaire » est paru en 1785 , où il s'agit maintenant de chercher les raisons de ce changement d'état d'esprit.

En 1785 comme en 1772, on calcule la probabilité d'un événement suivant le principe « abstrait » des cas également possibles qui en fait un objet mathématique, " mais on ne se borne pas à ce seul sens ", on "entend de plus» la probabilité en un «sens plus étendu »: " elle croît proportionnellement au motif de croire que l'événement arrivera $"$.

La locution « motif de croire " n'apparaît pas dans les manuscrits discutés précédemment ${ }^{77}$, elle présente donc un certain intérêt. Le " motif de croire » étant une notion première n'est défini que par son contexte. Si l'on considère un événement $\mathrm{A}$ dont l'arrivée est incertaine, on peut émettre le concernant deux " jugements ", « il arrivera plutôt que son contraire $\mathbf{N}$ » ou l'inverse. Le motif de croire que $A$ arrivera plutôt que $\mathrm{N}$ est ce qui nous "détermine " à choisir le premier jugement de préférence au second, l'épreuve des faits étant la sanction constante de ces «déterminations " par le bilan des jugements qui se sont avérés vrais ou faux.

Nous disposons donc de deux notions distinctes, la probabilité de nature abstraite qui compte les cas favorables ou non à l'événement $\mathrm{A}$ comme les boules blanches et noires d'un sac qui nous est donné dans les jeux de hasard ou qu'on reconstitue par la règle de CondorcetLaplace dans le cas des événements de la vie, et le motif de croire, lié à l'entendement humain, qui nous détermine à juger de l'arrivée de $\mathbf{A}$

75. Euvres..., op. cit. supra n. 55, t. I, p. 499.

76. Hist. Acad. Sciences pour 1781, Paris, 1784 , p. $707-728$; pour 1782, 1785, p. 674-691; pour 1783,1786 , p. 539-559; pour 1784, 1787, p. 454-468. On se reportera au premier article de P. CréPEL, cité supra $\mathrm{n}$. 50.

77. Il serait évidemment intéressant de dater précisément "motif de croire ", disons $1784 \pm 1$ avec probabilité $2 / 3$, en l'état actuel de nos motifs de croire. 
ou de son contraire $\mathrm{N}$. Condorcet se propose de montrer que celle-ci est proportionnelle à celle-là, " proposition " philosophico-mathématique dont la vérité dépend de la proposition préliminaire : « si la probabilité d'un événement $A$ est plus grande que celle de l'événement contradictoire $\mathrm{N}$, nous avons un motif de croire que l'événement $\mathrm{A}$ arrivera plutôt que de croire qu'il n'arrivera pas ${ }^{78}$. Si, en effet, on suppose la vérité de cette dernière proposition, celle de la première résulte, visiblement, du raisonnement suivant :

Plaçons-nous d'abord dans la situation d'un jeu de hasard, et supposons que $\mathrm{n}$ combinaisons produisent $\mathrm{A}$ et $\mathrm{m}$ son contraire $\mathrm{N}$. Par le théorème de Bernoulli, qui est une conséquence arithmétique certaine de la règle des combinaisons, on en déduit que si l'on répète un nombre suffisant de fois le jugement : « l'événement $A$ aura lieu plutôt que $\mathrm{N}$ », on aura une probabilité (mathématique) aussi proche de 1 qu'on le souhaite que « le rapport du nombre des jugements vrais à celui du nombre total des jugements sera entre $\frac{n+a}{n+m}$ et $\frac{n-a}{n+m}$, la quantité a étant une aussi petite quantité qu'on voudra ${ }^{79}$, et Condorcet conclut judicieusement :

"Si donc une probabilité très grande me donne un motif suffisant de croire que l'événement qui a cette probabilité arrivera, j'aurai un motif de croire que, dans un nombre très grand de jugements, le nombre de ceux oủ j'aurai rencontré la vérité, en jugeant que $\mathrm{A}$ doit arriver, sera au nombre de ceux où je me serai trompé, à peu près dans le même rapport que celui de $\frac{n}{n+m} \grave{a} \frac{m}{n+m}$. Je pourrai donc regarder le motif de juger que $A$ aura lieu, plutôt que de juger qu'il n'aura pas lieu, comme proportionnel à $\frac{\mathrm{n}}{\mathrm{m}+\mathrm{n}}$."

Si maintenant on quitte les jeux de hasard pour les événements de la vie, le raisonnement est identique, il suffit de remplacer la règle des cas également possibles par la règle de Condorcet (ou de Laplace ou de Bayes) et le théorème de Bernoulli par le théorème de Laplace ${ }^{80}$.

78. "Discours ", p. vii, et Élémens du calcul des probabilités, op. cit. supra n. 22, p. 81.

79. Élémens..., op. cit. supra, p. 82 ; nous suivons ici les Élémens qui sont plus pédagogiques que le « Discours » dont ils conservent le fond.

80. Guvres complètes, t. VIII, p. 33. Condorcet précise nettement que dans ce cas la probabilité calculée par sa règle n'est pas "réelle» mais «moyenne», ce qui ne l'empêche nullement, lorsque l'observation des événements passés peut être aussi prolongée qu'on le veut, de conduire à des " conclusions vraies " " dans un sens général », Elémens..., op. cit. supra n. 22 , p. $84-85$. On expliquerait cela maintenant en parlant de la « consistance " des procédures bayésiennes. 
Il ne reste plus qu'à justifier la proposition préliminaire ou simplement sa version affaiblie : " nous avons un motif de croire à la réalité d'un événement dont la probabilité est très grande et ne diffère de l'unité que d'une quantité aussi petite qu'on voudra ", qui suffirait à « assurer la vérité du sens plus étendu du mot probabilité ».

Ni le théorème de Bernoulli ni celui de Laplace ne sont plus d'un quelconque secours, les mathématiques ne pouvant sortir d'ellesmêmes.

Certes Bernoulli, sans autre forme de procès, avait identifié grande probabilité et certitude morale ${ }^{81}$ puis certitude morale et certitude absolue dans les choses de la vie ${ }^{82}$, ce qui lui avait permis d'étendre son théorème aux événements de la vie et d'en déduire notamment l'universel retour des choses. Mais Bernoulli n'avait pas publié son théorème et n'en avait développé aucune application, pas plus que ne l'avaient fait ses successeurs, à bien peu près.

On pouvait aussi ignorer superbement qu'il y avait là un problème, comme Laplace était en train de le faire, qui venait en 1780 et 1785 d'obtenir les premières applications véritables du calcul des probabilités à l'histoire naturelle de l'homme à l'aide du principe de Condorcet et de l'analyse moderne qu'il transformait par la même occasion. Pour montrer que le calcul des probabilités est applicable, pourquoi ne pas l'appliquer et de préférence aux questions les plus importantes de l'Astronomie physique, celles touchant à la correction des observations.

Mais les sciences les plus parfaites et les techniques les plus hautes ne sauraient accroitre le bonheur des hommes, ni leur servir de guides si l'on ne peut leur faire entendre les principes sur lesquels elles sont fondées ni leur montrer pourquoi elles ne trompent point ${ }^{83}$, a fortiori si l'on ne le sait pas soi-même.

La solution ne peut être scientifique, comme le reconnaîtra clairement Cournot qui s'est posé le même problème ${ }^{84}$ :

81. Ars conjectandi, op. cit. supra n. 25, p. 211.

82. Ibid., p. 217, " axiome IX».

83. CONDORCET, Bibliothèque de l'homme public, 1791, t. VII, p. 74. Sur ce point on lira Catherine KINTzLer, Condorcet, l'instruction publique et la naissance du citoyen, Paris, Gallimard (" Folio-Essais ", 55), 1987.

84. Exposition de la théorie des chances et des probabilités, Paris, Hachette, 1843, chap. IV, $\mathbf{n}^{\circ} 38$. Si on le désire, on pourra montrer, à titre d'exercice, que la théorie des chances et des probabilités de Cournot procède d'une démarche exactement parallèle à celle de Condorcet. Il suffit de substituer aux « motifs de croire » de Condorcet les " possibilites physiques " de Cournot et au principe de " constance des lois de la Nature " de Condorcet (ou de la "conscience du moi » de Turgot), celui de «l'impossibilité physique " de Cournot qui "opère " le même "passage ». 
" Pour opérer ce passage, de l'idée d'un rapport abstrait à celle d'une loi efficace dans l'ordre des réalités, et des phénomènes, les raisonnements mathématiques, appuyés sur une série d'identités, sont évidemment insuffisants. Il faut recourir à d'autres notions, à d'autres principes de connaissance; en un mot, il faut faire de la critique philosophique."

Il faut donc introduire un «principe philosophique de connaissance ", et tant qu'à faire celui qui sous-tend explicitement ou non toute la science et la philosophie des Lumières : " que la Nature suit des loix invariables, et que les phénomènes observés nous ont fait connaître ces loix ${ }^{85}$. Ce principe assure la liaison entre probabilité mathématique et motif de croire :

"supposons que d'un sac qui peut contenir des boules blanches et des boules noires, on ait tiré de suite un milliard de boules blanches, en remettant à chaque fois celle qu'on a tirée, pour que le rapport des boules blanches aux boules noires soit constant, et qu'on demande la probabilité de tirer une boule blanche de plus; cette probabilité sera exprimée par $1000000001 / 1000000002$. Or, il est aisé de voir que le motif de croire dans ce cas qu'on tirera encore une boule blanche, est précisément le même que celui qui nous porte à croire qu'un phénomène constamment observé se reproduira dans des circonstances semblables. Ce motif de croire qu'un événement très probable arrivera est donc le même que celui qui nous fait croire à la constance des lois de la nature $"{ }^{86}$.

Démonstration claire d'après ce qui précède, dont un exemple peut éventuellement concourir à rappeler le souvenir; une des lois constantes de la Nature, qui s'est manifestée avec le plus de régularité depuis l'origine, au moins sous nos latitudes, est celle qui assure le lever du soleil ; supposons avec Laplace (et contre Buffon) que : « l'on fasse remonter la plus ancienne époque de l'histoire à 5000 ans ou 1826213 jours " ${ }^{87}$, par la règle de Condorcet-Laplace la probabilité mathématique qu'il se lèvera encore demain est $\frac{\mathrm{n}+1}{\mathrm{n}+2}$, en posant $\mathrm{n}=$ 1826213 ; or il n'est pas de certitude plus assurée que celle-là, c'est donc qu'inversement si un événement a une probabilité, calculée suivant les mêmes règles, égale à cette même quantité $\frac{n+1}{n+2}$, nous avons

85. « Discours », p. x-xi.

86. Élémens..., op. cit. supra n. 22 , p. 85 . Rappelons que la formule $1000000001 / 1000000002$ résulte très simplement du principe de Condorcet-Laplace (cf. LAPLACE, 1774, Euvres complètes, op. cit. supra n. 58, t. VIII, p. 31).

87. P. S. LAPlace, Essai philosophique sur les probabilités, Paris, Bourgois, 1986, p. $45-46$. 
un motif de croire qu'il arrivera plutôt que son contraire équivalent à celui qui nous porte à juger que la terre et le soleil sont soumis à des lois invariables.

Ce n'est évidemment pas parce que la probabilité que le soleil se lèvera demain est très grande que nous croyons qu'il se lèvera demain, mais bien parce que nous croyons qu'il se lèvera demain et que sa probabilité mathématique se trouve être très grande que nous pouvons en conclure que « nous avons un motif de croire à la réalité d'un événement dont la probabilité est très grande $"$.

Le "passage " une fois assuré, tout le reste appartient aux domaines propres des mathématiques et de la philosophie.

Il reste, en effet, à intégrer le " principe de connaissance " des " lois invariables de la Nature » à une philosophie de la connaissance, notamment à celle de la sensation. Sans entrer dans un sujet fort bien traité ailleurs dans ce numéro, rappelons simplement que Condorcet considère le motif de croire dont il s'agit comme étant de même nature que celui qui nous porte à croire en l'existence des corps et plus fondamentalement encore, parce qu'il en dérive, que celui qui nous donne "conscience de notre moi ", puisque cette dernière naît de la " permanence », de la " perpétuité », de la " persistance ${ }^{88} \mathrm{du}$ moi, sensation première et répétée à tout instant, qui fonde ainsi non seulement toute la théorie de la connaissance mais encore l'applicabilité de la doctrine des hasards à l'évaluation de nos certitudes et à la conduite de la vie. Condorcet se recommande sur ce point de la philosophie de Turgot qui dans l'article « Existence » de l'Encyclopédie ${ }^{89}$

" cherche [...] quel est le véritable sens que nous attachons à ce mot et trouve que l'Existence est pour nous l'idée de la permanence de certaines collections de sensations, qui, dans des circonstances semblables reparaissent constamment les mêmes ou avec des changements assujettis à certaines loix. Quand nous disons qu'un objet existe, nous entendons seulement qu'un système de sensations simultanées ayant été aperçu par nous pendant une certaine durée, ayant disparu plus d'une fois et s'étant représenté encore, nous sommes portés, même lorsque ce système de sensations cesse de s'offrir à nous, à regarder ce même système comme devant se présenter de nouveau de la même manière si nous nous retrouvions dans les mêmes circonstances et nous disons alors que cet objet existe.

88. Par ex., “ Sur la persistance de l'âme », VII, ms. 885 (I-II), ff. 245-262, reproduit in CondorCet, Sur les élections, Paris, Fayard, 1986, p. 627-641 et en partie in R. RASHED, op. cit. supra n. 50, p. 134-136. Je tiens cette référence de $P$. Crépel que je remercie. Si ce texte était daté, il permettrait peut-être d'éclaircir un peu la chronologie du parcours probabiliste de Condorcet; on y trouve exposée la théorie du motif de croire de Condorcet sur laquelle on se reportera au livre de R. RASHED, op. cit. supra, chap. III.

89. Encyclopédie, t. VI, 1756. 
Cette théorie, si neuve qu'à peine fut-elle entendue de quelques philosophes, avait des conséquences importantes : elle était liée avec la théorie entière de la nature de nos connaissances, et de celle de l'espèce de certitude à laquelle nous pouvons atteindre. C'était un grand pas dans la connaissance la plus intime de l'esprit humain, et presque le seul qu'on ait fait depuis Locke $"{ }^{90}$.

\section{III. - CONCLUSION}

La boucle est bouclée, " les vérités des sciences morales et politiques sont susceptibles de la même certitude que celles qui forment le système des sciences physiques ", parce qu'elles sont toutes ultimement fondées sur la conscience du moi qui résulte de la permanence de nos sensations.

Mises à part les certitudes absolues des identités mathématiques ou métaphysiques dont on a une conscience immédiate, toutes nos certitudes naissent du plus ou moins de permanence des observations. Certes, il existe des vérités qui apparaissent comme certaines alors qu'un petit nombre seulement d'observations les a confirmées et parfois même une seule, voire aucune, mais c'est parce que ces vérités appartiennent à une classe de vérités dont on a déjà éprouvé la permanence $^{91}$. La certitude mathématique elle-même, lorsque l'on a cessé

90. Vie de M. Turgot, Londres, 1786, p. 21-22 (cf. aussi, p. 210-215). On sent dans ce texte l'enthousiasme d'un néophyte, c'est donc une observation supplémentaire qui vient renforcer la probabilité de la datation de la doctrine des motifs de croire de Condorcet, proposée note 77 , que l'on peut maintenant considérer comme se situant dans l'intervalle $1784 \pm 1$ avec probabilité 3/4, (suivant la règle de Condorcet-Laplace $n+1 / n+2$ ), tout en faisant observer qu'il ne s'agit là que d'une probabilité moyenne et même très moyenne puisqu'elle ne prend en compte que deux observations, le " Discours" et la "Vie de Monsieur Turgot».

La philosophie de Turgot ne semble pas avoir suscité beaucoup d'études, Victor Cousin lui consacra la XVIIe leçon de son cours de l'année universitaire 1815-1816, alors qu'il suppléait Royer-Collard (nommé au Conseil royal de l'Instruction publique) à la chaire d'Histoire de la philosophie moderne de la Faculté des lettres de Paris. Cf. Victor Cousin, Cours de l'histoire de la philosophie moderne, Paris, 1846, 1re série, t. I, p. 147-150. Cousin semble suivre Condorcet : « En somme l'idée d'existence est pour Turgot une idée abstraite et générale dont l'origine est dans la conscience du moi. L'existence personnelle est attestée directement par la conscience. C'est là qu'est la première réalité, et toutes les autres ne sont que des inductions de celle-là : opinion très considérable, qui tranche avec la plupart des opinions du temps. Partout Turgot répète cette expression de conscience et de sentiment du moi, qu'on trouverait en vain dans les ouvrages de Condillac. » Mais l'on ne peut se fier à Victor Cousin.

91. Ce point, traité par Condorcet dans son « Mémoire sur le calcul des probabilités » peut servir de fondement au déterminisme ou plutôt au "comportement déterministe » où l'on fait " comme si ", comme l'a très bien vu Bruno DE FINETTI, par ex., Actualités scientifiques et industrielles, 734, 1938, p. 18. 
d'avoir présente à l'esprit " l'évidence intuitive " des vérités intermédiaires qui l'assurent et dont on ne conserve que le souvenir, est fondée sur "l'expérience constante » que les souvenirs ne trompent pas en de telles circonstances ${ }^{92}$. Quant aux principes simples et clairs des sciences physiques: "l'expérience constante que les faits sont conformes à de certains principes est pour nous le seul motif de les croire. "

Or le calcul mathématique des probabilités nous apprend à déterminer, selon des règles fixes, la probabilité qu'un événement qui s'est toujours produit se produira encore et cette probabilité est d'autant plus grande que l'observation s'est prolongée plus longtemps et elle est immense dans le cas de ces perpétuités qui assurent nos certitudes, elle correspond à la probabilité de tirer une boule blanche d'un sac qui en contiendrait un nombre énorme et une seule boule noire ${ }^{93}$. C'est donc qu'un homme placé devant un sac de cette composition-là a un motif de croire qu'il va tirer une boule blanche, de même nature que celui qui lui fait juger de l'existence des corps et prendre conscience de lui ${ }^{94}$. Du même coup, les théorèmes arithmétiques usuels donnent une valeur pratique au calcul des probabilités : la probabilité d'un événement est, avec certitude, du moins avec la seule certitude à laquelle nous puissions atteindre, à très peu près égale au " rapport des jugements vrais que j'émettrais » (en prononçant qu'il arrivera plutôt que son contraire) au nombre total des jugements ${ }^{95}$.

Même s'il est encore dans l'enfance ${ }^{96}$, le calcul des probabilités peut alors, avec certitude, être un guide de la vie, notamment en indiquant le degré de vraisemblance de l'Arithmétique politique, ces statistiques sociales ou morales des $X V I I^{e}$ et $X V I I^{e}$ siècles qui ne savaient que se

92. «Discours", p. xiii.

93. Même si cette « correspondance » n'est que moyenne et ne devient réelle que pour les nombres immenses, ce qui est précisément le cas lorsqu'il y a permanence.

94. On peut naturellement contester ce point de rencontre entre la théorie mathématique des probabilités et la théorie philosophique de la connaissance, qui semblent s'appuyer l'une sur l'autre comme deux chaises bancales, mais il faut s'efforcer d'en saisir l'aspect dynamique qui assure la perfectibilité des connaissances et éventuellement des sociétés humaines.

95. Cournot, op. cit. supra n. 84, reprend exactement la même expression (cf. no 93, 107,111 ). C'est, en effet, le «criterium » des énoncés probabilisés qui fonde ses applications. Citons, par ex., Jerzy Neyman, Annals of Stat., vol. 9, 1, 1981, p. 1 : « According to Borel, the reliability of probabilistic test criteria must be measured by the long-range frequency of correct conclusions that a given criterion can provide for judging the hypotheses tested. The reader will notice that this statement of Borel, somewhat vague as it is, represents the basic idea of the modern theory of testing hypotheses. " Cf. Émile BorEL, Valeur pratique et philosophie des probabilités, Paris, Gauthier-Villars, 2e éd., 1952, p. 90.

96. Par ex., note de Condorcet sur la chaire d' "Application du calcul aux sciences morales et politiques » de la première classe des lycées, P.V. du Comité d'Inst. publ. de l'Assemblée législative, éd. par James Gulllaume, Paris, 1889, p. 236. 
contredire ou se payer de chiffres ${ }^{97}$. Il peut aussi réduire en nombres " la probabilité des décisions rendues à la pluralité des voix » et l'on est revenu au point de départ. Faire du calcul des probabilités, même si l'on se borne à raisonner à l'intérieur des mathématiques les plus abstraites, comme le fera Condorcet, c'est de toutes les façons possibles contribuer à la "science du bonheur public" .

Il faudrait discuter maintenant du destin de la doctrine des probabilités de Condorcet. On montrerait que, mise à part une courte période d'euphorie après Thermidor où l'on tenta de l'expliquer aux élèves de l'École Normale de l'an III ${ }^{98}$, elle n'eut que peu de postérité immédiate, Laplace oubliant de la mentionner, bien qu'on puisse la lire parfois dans l'Essai philosophique ${ }^{99}$, Lacroix, le disciple officiel de Condorcet, en présentant une version peu reconnaissable plus inspirée de Hume et de Laplace ${ }^{100}$. Poisson ${ }^{101}$, pour sa part, avait peu de goût pour les questions de fondements, ce qui du reste lui fit grand tort, et Cournot ${ }^{102}$ développa de son côté une philosophie diamétralement opposée, bien que symétrique à bien des égards.

On montrerait ensuite qu'elle est réapparue au début du $\mathrm{xx}^{\mathrm{e}}$ siècle sous des formes diverses ${ }^{103}$ et qu'elle imprègne encore toute la statistique mathématique actuelle.

La raison en est qu'elle n'impose ni de croire absolument en l'existence de probabilités dans la nature des choses ${ }^{104}$, comme l'exigeait la philosophie janséniste de Cournot, ni de croire au principe de Raison suffisante, comme Laplace s'était finalement décidé à le faire ${ }^{105}$. Elle n'oblige pas 'non plus à se déclarer "subjectiviste " puisqu'elle croit en la certitude de ses résultats, mais elle ne rejette pas non plus ceux qui ne voient pas comment ne pas l'être. Elle invite simplement la " philosophie à s'unir aux sciences et surtout aux sciences de calcul $\gg{ }^{106}$.

97. Cf. Condorcer, complément de l'article «Arithmétique politique » de l'Encyclopédie méthodique, Paris, 1784, I.

98. Ce devait être l'intention de Volney et surtout de Garat, on se reportera à leurs leçons dans les volumes des Séances des écoles normales, Paris, an IV.

99. Essai philosophique sur le calcul des probabilités, 1 re éd. 1814,5 e éd. 1825 , rééd. en 1986, par C. Bourgois, Paris.

100. S.F. LACROIX, op. cit. supra n. 16.

101. Simeon D. Poisson, Recherches sur la probabilité des jugements..., Paris, Bachelier, 1837.

102. A. A. Cournot, Exposition de la théorie des chances..., Paris, Hachette, 1843.

103. Op. cit. supra n. 95.

104. Bien que Condorcet, comme Laplace, ait eu quelque motif d'y croire.

105. Op. cit. supra n. 87.

106. «Discours », p. clxxxvii. 
«Alors la précision des résultats, et leur certitude, marquerait une limite bien prononcée entre les opinions spécieuses, qui ne sont que les aperçus d'un premier coup d'œil, et celles qui méritent d'être mises au rang des vérités qu'on doit suivre dans la pratique ${ }^{107}$.

Les précisions du calcul et les certitudes de la philosophie rétablissent l'homme "dans les droits comme dans la dignité de sa nature ", alors « il existe véritablement avec ses semblables, dans un élysée que sa raison a su se créer et que son amour pour l'humanité embellit des plus pures jouissances $» 108$.

\section{Bernard BRU, Université Paris VI.}

107. Ibid. On croirait lire « l'adresse présidentielle » d'un "Congrès de statistique " contemporain, par ex. celle prononcée à la fin de l'année 1986 devant l'Association américaine de statistique par son président en exercice D. W. Marquardt (E. I. du Pont de Nemours and Company) qui conclut : "Applied statisticians, theoretical statisticians, and philosophers can make the journey together cooperatively, with far better results than if they attempt their journeys separately, toward separate directions " (Journal Amer. Stat. Assoc., vol. 82, 397, 1987, p. 6).

108. Esquisse..., op. cit. supra n. 1, dernière phrase. 